\title{
TRANSLATED ARTICLE
}

\section{Relationship between the Shape and Spacing of Convex Points and the Roughness Sensation in a Dot Pattern Texture}

\author{
Junpei AIZAWA* and Masayoshi KAMIJO** \\ * Nagano Prefecture General Industrial Technology Center, 1-18-1 Wakasato, Nagano-shi, Nagano 380-0928, Japan \\ ** Shinshu University, 3-15-1 Tokida, Ueda-shi, Nagano 386-8567, Japan
}

\begin{abstract}
The spacing and curvature of dots are assumed to be factors determining the roughness sensation of a dot pattern. We investigated the relationship between these two parameters and the roughness sensation. We used a three-dimensional printer to produce samples with different combinations of curvature and spacing, and conducted a sensory evaluation to select combinations presenting the same sense of roughness from among them. Experimental results showed that the contour lines of the same sense of roughness are concave and monotone decreasing on the dot curvature versus dot spacing plane. It was found that the roughness sensation is proportional to the logarithm of dot curvature, adopting a previously obtained result that the roughness sensation is proportional to the logarithmic value of the dot spacing. The experiment also revealed non-negligible differences in contour lines among participants and possibility of sorting them into three groups with different roughness perception.
\end{abstract}

Keywords: Texture design, Roughness sensation, Curvature

\section{INTRODUCTION}

The texture of a surface is accepted through visual and tactile senses and is an important factor determining the impression of a product. In this paper, the roughness sensation obtained through tactile sensing is studied because of its importance. We create a variety of dot patterns with different combinations of dot shape (curvature) and dot spacing on test surfaces using a three-dimensional (3D) printer. By searching for combinations presenting the same roughness sensation among these patterns, we find a pattern of contour lines of the roughness feeling in the twodimensional space of the dot curvature and dot spacing, and from this, we estimate the relationship between the two parameters and the roughness sensation. Adopting this method, we present a quantitative relationship between the feeling of roughness and the dot spacing in the range of $0.4-0.9 \mathrm{~mm}$ and dot curvature in the range of $2-8 \mathrm{~mm}^{-1}$.

\section{BACKGROUND}

Because resin products made by injection molding do not have specific textures derived from materials or processing methods, including geometric patterns and leather patterns, these patterns are often artificially imparted by emboss processing or painting. In emboss processing, the texture pattern is usually transferred to

This article is a translated version of the original paper published in "Transactions of Japan Society of Kansei Engineering," Vol.18, No.3, pp.171-178. the mold by etching. It has recently become possible to control the fine shape employing advanced processing methods, such as the digital shibo process (i.e. digitized embossing process) involving microfabrication and multilayer etching. Specific design guidelines, based on these processing techniques, are required to control the tactile sensation according to the design value of the texture.

Okamoto [1] reviewed the literature and revealed the hierarchical structure of the sense from the physical characteristics of the material to the impression of higher order is determined. The process of determining the impression of an object by tactile sensation is hierarchical in that there is a layer of perception of the material at the lowest level, a layer of sensibility on that, and a layer of preference thereon. An impression is drawn on this basis. The tactile perception of the material of the lowermost layer is expressed in a five-dimensional space that includes microscale roughness sensation, macroscale roughness sensation, hardness sensation, friction sensation, and thermal sensation. According to this structure, to control the impression of the product by the shape of the texture, it is necessary to know how the physical dimension of the texture works in the primitive five-dimensional tactile space.

The following examples are given as recent examples of controlling the tactile sensation by the shape of the texture from the viewpoint of developing a resin product. Ban et al. [2] developed a soft feel controlled by embossing, Yonehara et al. [3] showed the effect of gradational patterns on the positional accuracy of active touch, and Kawasegi et al. [4-6] expressed texture using microfabricated molds. However, few studies have presented specific dimensional values. 
Aizawa et al. investigated the relationship between the roughness sensation and the dimensional parameters for a dot pattern, in which hemispherical convex points are arranged in a lattice pattern on the surface. They found that the determining factors of roughness sensation are different between the visual and tactile; that is, the size of the dots in the visual and the dot spacing in the tactile [7]. They also showed that a difference in dot height is perceived as a difference in roughness only under the condition that the skin is in contact with the plane between the dots, and they gave the dimensional conditions under which the difference is perceived [8].

The perception of the roughness sensation is divided into a macroscale roughness perception relating to the pressure distribution of the contact surface without requiring a touch motion and a microscale roughness perception relating to vibration on the skin surface with a touch motion. The macroscale and microscale roughness perceptions are distinguished by the period of the stimulus, and the boundary is believed to be several hundred micrometers to $1 \mathrm{~mm}$ [9]. In the case of a dot pattern with spacing of $1 \mathrm{~mm}$ or less, which is the target pattern in this paper, it is considered that microscale and macroscale perceptions of roughness coexist. It is thought that the roughness feeling is judged microscopically by the amplitude and frequency of skin vibration and macroscopically by the magnitude of pressure generated on the skin and its distribution density. In either case (if the dot height can be ignored), the shape of the dots and the dot spacing might be determining factors.

The "roughness" that we evaluate as one tactile sense can be thought of as the weighted combination of two evaluations. In this paper, we investigate the relationship between two-dimensional parameters and the roughness sensation and examine how to determine the roughness sensation.

As an example, focusing on a multidimensional structure that constitutes a sensation of roughness, Noro et al. $[10,11]$ conducted a sensory evaluation of 18 types of turningprocessed sample with different values for four physical quantities, namely the surface roughness, pitch, undulation, and tip angle. By applying a multidimensional scale construction method to the results, they revealed the structure that the roughness sensation is determined from physical quantities. Mishima et al. [12] similarly conducted a sensory evaluation of a turning surface for varying surface roughness $(\mathrm{Rz})$ and pitch, revealed that the roughness sensation increased with both $\mathrm{Rz}$ and pitch, and found that the participants in their study could be divided into those affected by $\mathrm{Rz}$ and those affected by the pitch. They also presented the pitch change corresponding to the change in Rz.

\section{METHOD}

We used a three-dimensional printer to produce samples with different combinations of curvature and spacing, and conducted a sensory evaluation to select combinations presenting the same sense of roughness from among them.

\subsection{Model of the roughness sensation of a dot pattern}

Previous research [8] has shown that the roughness sensation increases as the dot spacing increases. In addition, it is obvious that the roughness sensation increases as the dot shape sharpens. Figure 1 shows that, from condition $\mathrm{O}$, the roughness sensation increases as the dot spacing (A) lengthens or the dot shape (B) sharpens. Conversely, the roughness sensation decreases as the dot spacing narrows (a) or the dot shape becomes blunter (b). There are therefore combinations presenting a roughness sensation equivalent to that of the original condition $\mathrm{O}$ in the case of extending the dot spacing and blunting the dot shape $(\mathrm{Ab})$ and in the case of narrowing the dot spacing and sharpening the dot shape $(\mathrm{aB})$. If we can search for combinations presenting an equivalent roughness sensation, we can draw contour lines of the roughness sensation in the two-dimensional parameter space of dot spacing and dot curvature as shown in Figure 2.

A contour in Figure 2 represents how the two parameters are evaluated with weighting in the course of judging the roughness sensation. The contour also clarifies the relationship between dot shape and roughness sensation.

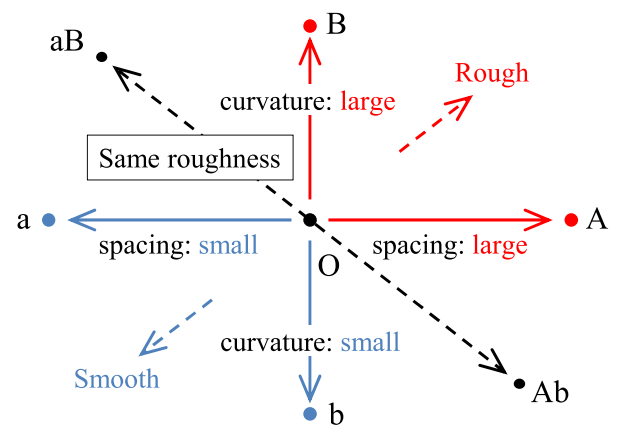

Figure 1: Relationship between changes in dimensional parameters and the change in the roughness sensation

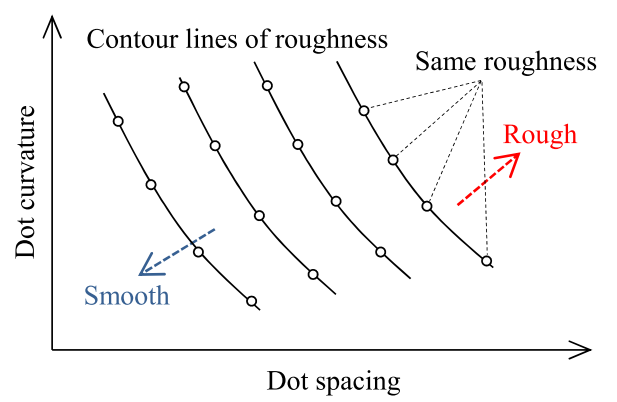

Figure 2: Contour lines of roughness sensation in two-dimensional parameter space 


\subsection{Sensory evaluation experiment}

To obtain the relationship between the dimensions and sensation, it is necessary to comprehensively search the objective dimensional region. However, it is practically difficult to measure the sensation value for many types of samples through paired comparisons.

Therefore, to clarify the overall picture more easily, we drew contour lines of the roughness sensation by selecting a dot spacing/dot curvature combination having the same roughness sensation from various types of sample made with a $3 \mathrm{D}$ printer.

(1) Search for contour lines of the roughness sensation

Figure 3 illustrates the preparation of four samples having constant curvature and different spacings as reference samples. In addition, a comparison target group (target samples) having a curvature different from that of the reference sample was prepared. In the experiments, participants were asked to choose the target samples that presented a roughness sensation that was the same as that of the reference sample. Four sets of target samples were made with different curvatures; these are referred to as series A-D. There are five levels of curvature when including the reference sample, and a set of five dot spacings/curvatures on a contour line is thus obtained. Four contour lines are obtained for four reference samples having different roughness sensations. Adopting this method, the search range can be expanded easily by expanding the range of the dot spacing of the reference sample and dot curvature of the target sample.

\section{(2) Method}

Four reference samples were arranged in front of a participant in order of dot spacing with roughness increasing from left to right. Similarly, target samples were arranged in order of dot spacing before the reference sample with roughness increasing from left to right. Participants touched and compared samples and stated which of the target samples presented the same roughness sensation as each reference sample. Answers intermediate of two target samples were also allowed. This procedure was repeated four times from series A to series D. Participants were instructed to touch laterally with the

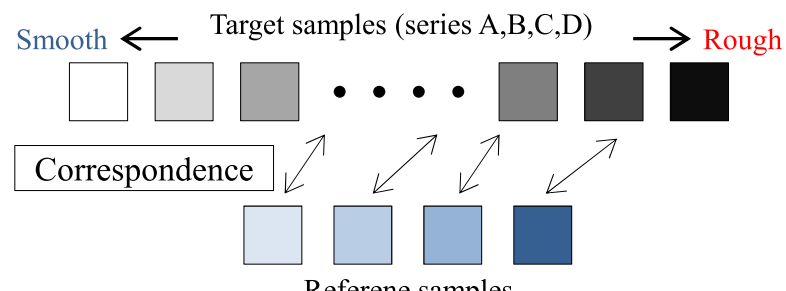

Figure 3: Sensory evaluation method right index finger. The touching frequency, contact force, and time taken were left as free parameters.

The experimental time was about 5-15 minutes per participant. Participants were 23 men and 7 women in their twenties and fifties.

The visual features of a texture may affect the evaluation of the roughness sensation when a sample is touched and viewed at the same time. Yanagisawa et al. [13,14] quantitatively evaluated the expected effect of visual perception on roughness in experiments, in which the tactile sensation was evaluated while images of different textures on the sample surface were synthesized. The experiment showed that increasing the difference between visual prediction and tactile sensation emphasizes the roughness sensation. We assumed a general consumer product that the user will touch while looking at the surface, and we therefore did not restrict the vision of the participants.

\subsection{Samples}

We create a variety of dot patterns with different combinations of dotcurvature and dot spacing on test surfaces using a 3D printer.

(1) Principle of creating a dot shape

Dot pattern samples were fabricated using an inkjet 3D printer. When making minute convex points using an inkjet 3D printer, even if the design data are rectangular parallelepipeds, the modeled shape becomes a gentle hill approximating a hemisphere. Figure 4 shows an example of the shape when fine parallelepiped data are modeled. Aizawa et al. [15] conducted a modeling experiment for many small parallelepipeds and found that the shape of modeling can be predicted by assuming a numerical model that considers variations in landing points of ink in the lamination process. By applying this property, it is possible to create smooth dots whose curvature and height are controlled within a dimensional range of several times to several tens of times the modeling resolution of the printer. We previously confirmed that the dot shape created adopting this method has a highly reproducible shape, sufficient for practical sensory evaluation.
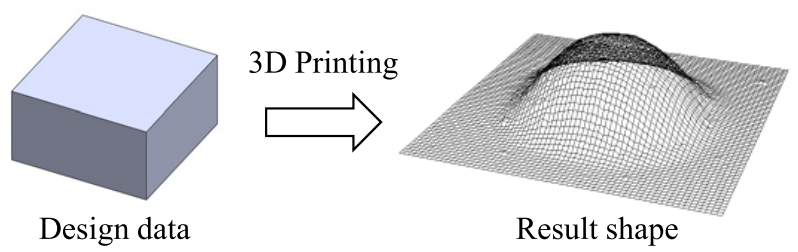

Figure 4: Design data and modeling result when using a $3 \mathrm{D}$ printer 
Table 1: Dimensional parameters of dot patterns (design values)

\begin{tabular}{|c|c|c|c|c|c|c|c|c|c|c|c|c|c|c|}
\hline & $\begin{array}{c}\text { Dot width } \\
(\mathrm{mm})\end{array}$ & 0.38 & 0.42 & 0.47 & 0.51 & 0.55 & 0.59 & 0.63 & 0.68 & 0.72 & 0.76 & 0.8 & 0.85 & 0.89 \\
\hline series A & 0.17 & $\mathrm{~A}_{9}$ & $\mathrm{~A}_{10}$ & $\mathrm{~A}_{11}$ & $\mathrm{~A}_{12}$ & $\mathrm{~A}_{13}$ & $\mathrm{~A}_{14}$ & $\mathrm{~A}_{15}$ & $\mathrm{~A}_{16}$ & $\mathrm{~A}_{17}$ & $\mathrm{~A}_{18}$ & $\mathrm{~A}_{19}$ & $\mathrm{~A}_{20}$ & \\
\hline series B & 0.25 & & $\mathrm{~B}_{10}$ & $\mathrm{~B}_{11}$ & $\mathrm{~B}_{12}$ & $\mathrm{~B}_{13}$ & $\mathrm{~B}_{14}$ & $\mathrm{~B}_{15}$ & $\mathrm{~B}_{16}$ & $\mathrm{~B}_{17}$ & $\mathrm{~B}_{18}$ & $\mathrm{~B}_{19}$ & $\mathrm{~B}_{20}$ & \\
\hline Reference & 0.34 & & & & $\mathrm{R}_{12}$ & & $\mathrm{R}_{14}$ & & $\mathrm{R}_{16}$ & & $\mathrm{R}_{18}$ & & & \\
\hline series C & 0.42 & & & & $\mathrm{C}_{12}$ & $\mathrm{C}_{13}$ & $\mathrm{C}_{14}$ & $\mathrm{C}_{15}$ & $\mathrm{C}_{16}$ & $\mathrm{C}_{17}$ & $\mathrm{C}_{18}$ & $\mathrm{C}_{19}$ & $\mathrm{C}_{20}$ & \\
\hline series D & 0.51 & & & & & & $\mathrm{D}_{14}$ & $\mathrm{D}_{15}$ & $\mathrm{D}_{16}$ & $\mathrm{D}_{17}$ & $\mathrm{D}_{18}$ & $\mathrm{D}_{19}$ & $\mathrm{D}_{20}$ & $\mathrm{D}_{21}$ \\
\hline
\end{tabular}

\section{(2) Dimensional parameters of the dot pattern}

Dots were designed as rectangular parallelepipeds whose widths were an integral multiple of the printer resolution (horizontal $0.0423 \mathrm{~mm}$, height $0.016 \mathrm{~mm}$ ). The dot curvature was determined by the width of the rectangular parallelepiped. The dot height was designed so that the height after modeling was $0.16 \mathrm{~mm}$ and the skin of the finger did not come into contact with the plane between dots. The dot spacing was also set to an integral multiple of the printer resolution. Reference samples $R_{12}, R_{14}, R_{16}$, and $\mathrm{R}_{18}$ respectively had dot spacing of $12,14,16$, and 18 times the printer resolution and a data width of 8 times $(0.338 \mathrm{~mm})$ the printer resolution. The data width of the target sample was respectively 4, 6, 10, and 12 times the printer resolution. Small dot spacing resulted in lower curvature of the dot under the effect of the adjacent dots, and the smallest possible dot spacing was restricted by the data width. Under this modeling condition, the collapse of the shape was greater when the gap between data was $0.085 \mathrm{~mm}$ or less. Table 1 gives the design values of the dot patterns used in the experiment.

Each sample was a plate having an area of $48 \mathrm{~mm}$ square and a thickness of $2.5 \mathrm{~mm}$, and a dot pattern was formed in the central area of $22 \mathrm{~mm} \times 34 \mathrm{~mm}$.

(3) Modeling conditions

Each sample was shaped under the condition that the surface was on the top and the support material was not attached to the surface. The main specifications and materials of the 3D printer are given below.

- Model: Stratasys EDEN 260 VS

- Resolution X and Y axes: 600 dpi (0.0423 mm), $\mathrm{Z}$ axis: $1600 \mathrm{dpi}(0.016 \mathrm{~mm})$

- Resin: VeroWhitePlus RDG 38 (hard acrylic type)

\section{RESULTS AND DISCUSSION}

The contour lines of roughness sensation was obtained by the experiment, and the relationship between the dimensional parameter and the roughness sensation was clarified by the contour lines. The equation for estimating the magnitude of the roughness sensation from the dot spacing and dot curvature was formulated, and the individual differences were also examined.
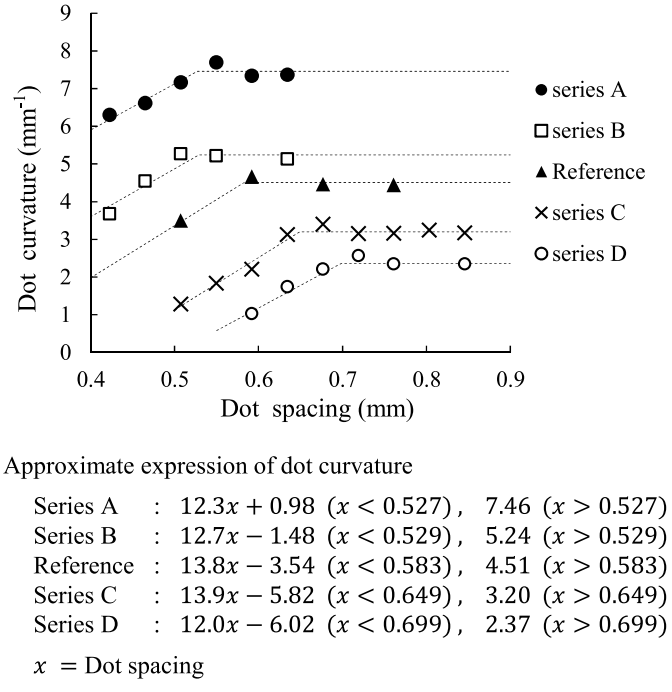

Figure 5: Measurements of sample dimensions

\subsection{Sample shape}

Figure 5 shows the curvature of the top of the dot (about one-third of the diameter) measured with a laser microscope (OLS-4100, OLYMPUS Corp.). When the dot spacing is narrow, the curvature decreases under the influence of adjacent dots. Because this effect appears linearly with respect to the dot spacing, the curvature is deemed to follow a linear approximation formula in this range. For the range not affected by adjacent dots, the curvature was approximated as the average value. We use the value obtained adopting this approximation as the curvature of each series of sample in this paper.

\subsection{Sensory evaluation results}

Figure 6 shows the average values of sensory evaluation results for the 30 participants. The results are the average values of the dot spacing for each target sample whose roughness sensation is equal to that of the reference sample. A smaller spacing was chosen for series A and $B$ with curvature larger than that of the reference sample while a larger spacing was chosen for series $\mathrm{C}$ and $\mathrm{D}$ with curvature smaller than that of the reference sample. As expected in Section 2.1, the change in dot curvature was compensated for by the change in dot spacing such that the same roughness sensation was obtained. 


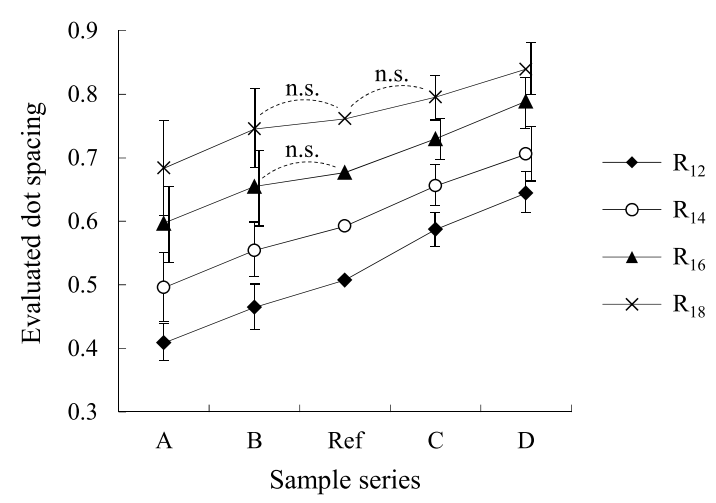

Figure 6: Series of samples and evaluated dot spacing

To test whether there is a significant difference between the average values of the five groups of series A to D and the reference sample, a multiple comparison test was conducted among the five groups for each dot spacing. A significant difference $(\mathrm{P}<0.01)$ was observed among all groups except $(\mathrm{P}<0.05)$ in the three comparisons indicated "n.s." in Figure 6.

\subsection{Contour lines}

We drew contour lines of the roughness sensation on the plane of dot spacing and dot curvature and investigated the distribution of the roughness sensation.

(1) Contour lines of average values

The results of the previous section are shown in the scatter diagram of the dot curvature versus dot spacing shown in Figure 7a. The contour lines are curved, being steep for large curvature and shallow for small curvature. It is considered that there was no significant difference for three pairs in the multiple comparison test because they are in a region where the contour lines are steep and the curvature does not change greatly.

When logarithmic values are taken for both axes as shown in Figure $7 b$, the points on each contour line are almost linearly aligned, and the first-order approximate expression is highly correlated with the experimental value. Expressions approximating the curves are given in the figure.

(2) Quantification of contour lines

Although the form of the contour line was shown in the previous section, we cannot find the three-dimensional distribution unless the magnitude of the roughness sensation (i.e., the height of the contour line) indicated by each contour line is known. Previous research [8] has shown that, in the evaluation of roughness through paired comparison for a dot pattern having a dot spacing of 0.4-0.8 $\mathrm{mm}$ and constant dot curvature, the roughness sensation tends to be proportional to the logarithmic value of the dot spacing. With reference to this finding, assuming that the roughness sensation is proportional to
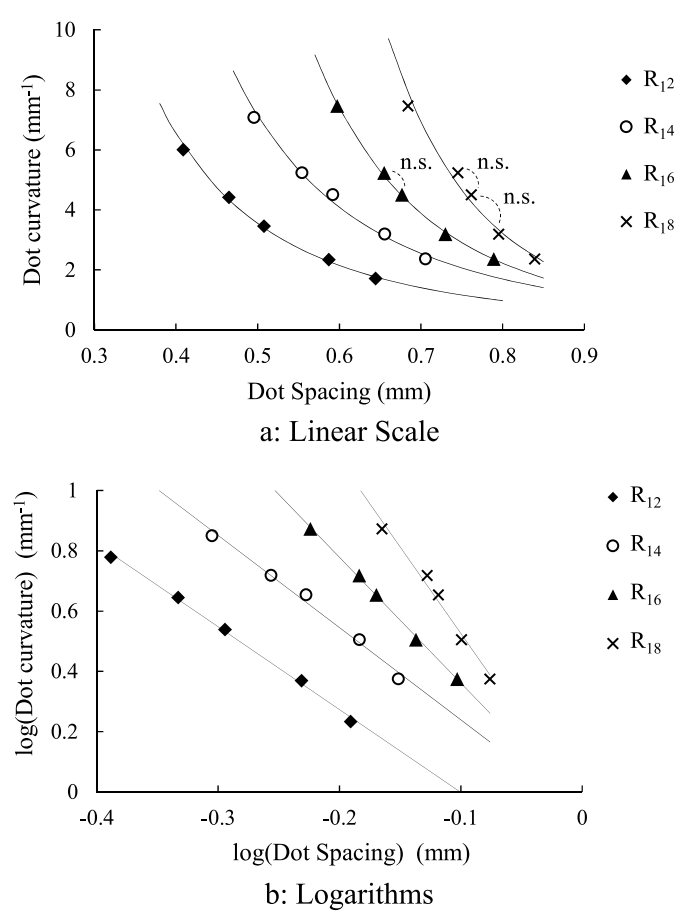

$$
\begin{aligned}
& \text { Approximate expression by logarithmic approximation } \\
& \mathrm{R}_{12} \quad: \log (y)=-2.75 \log (x)-0.28 \quad \mathrm{R}^{2}=0.997 \\
& \mathrm{R}_{14}: \log (y)=-3.06 \log (x)-0.07 \quad \mathrm{R}^{2}=0.989 \\
& \mathrm{R}_{16}: \log (y)=-4.18 \log (x)-0.06 \quad \mathrm{R}^{2}=0.999 \\
& \mathrm{R}_{18} \quad: \log (y)=-5.74 \log (x)-0.05 \quad \mathrm{R}^{2}=0.981 \\
& x=\text { Dot spacing, } y=\text { Dot curvature }
\end{aligned}
$$

Figure 7: Contour lines of roughness and approximate expressions given as exponential functions

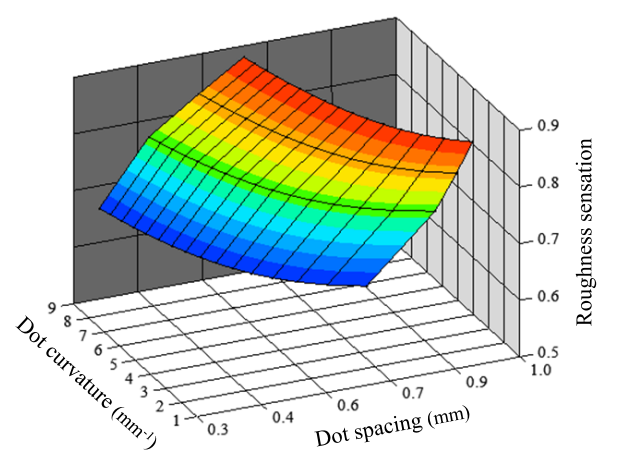

Figure 8: 3D distribution of the roughness sensation drawn from contour lines

the logarithmic value of the dot spacing in the reference sample of this paper also, the magnitude of the roughness sensation of the four contour lines $R_{12}$ to $R_{18}$ can be determined from the dot spacing of the reference sample. We therefore quantified the value of each contour line using the logarithmic value of the dot spacing at the curvature of the reference sample $\left(4.51 \mathrm{~mm}^{-1}\right)$. (The dot spacing was multiplied by 10 for convenience.)

The distribution of the roughness sensation quantified as described above is represented three-dimensionally in Figure 8. For more accurate quantification, it is desirable to determine the roughness sensation of the reference sample through paired comparison. 


\subsection{Relationship between dimensional parameters and roughness sensation}

We investigated the relationship between individual parameters and roughness sensation.

(1) Relationship between the dot spacing and roughness sensation

The relationship between the dot spacing and roughness sensation by curvature is shown in Figure 9. The dot spacing on the horizontal axis is logarithmic. Even for samples with curvatures different from those of the reference samples, the roughness feeling was almost linearly related to the logarithmic value of the dot spacing.

(2) Relationship between the dot curvature and roughness sensation

The relationship between the dot curvature and roughness sensation by dot spacing is shown in Figure 10. The dot curvature on the horizontal axis is logarithmic. As with the dot spacing, the roughness sensation was almost linearly related to the logarithmic value. It is thus expected that the roughness feeling is proportional to the logarithm of the dot curvature when the dot spacing is constant.

\subsection{Formula for estimating the magnitude of the roughness sensation}

On the basis of the results of quantification in the previous section, it is expected that the curved surface in Figure 8 will be close to a flat surface in a three-dimensional space with dot spacing (logarithm), dot curvature (logarithm), and roughness sensation as the three axes. The roughness sensation can therefore be estimated from the dot spacing and the dot curvature via a function representing this approximate plane.

In the plane, $\mathrm{z}$ is expressed in the form of a linear sum of $\mathrm{x}$ and $\mathrm{y}$. Multiple regression analysis was therefore applied to the experimental values of the dot spacing (logarithm) and dot curvature (logarithm), giving the estimation formula (1) of the roughness sensation.

$$
\begin{aligned}
& \text { Roughness sensation } \\
& =1.06 \times \log (\text { Dot spacing })+0.30 \times \log (\text { Dot curvature })+0.82
\end{aligned}
$$

In Equation (1), if the roughness sensation is a constant, the equation becomes an expression representing a contour line. Figure 11 shows the experimental value and contour lines drawn from the estimation equation. Although they almost agree with each other, there are errors in the estimation result particularly for $\mathrm{R}_{18}$. These errors represent the deviation of the experimental value with respect to the plane fitted by the estimation formula. According to Equation (1), the experimental

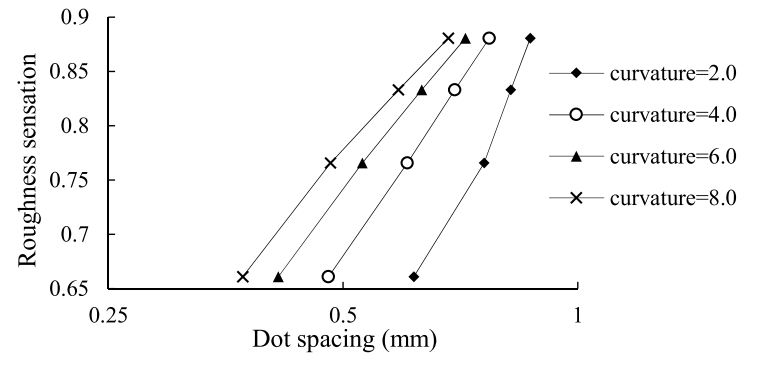

Figure 9: Relationship between the dot spacing and roughness sensation expected from contour lines

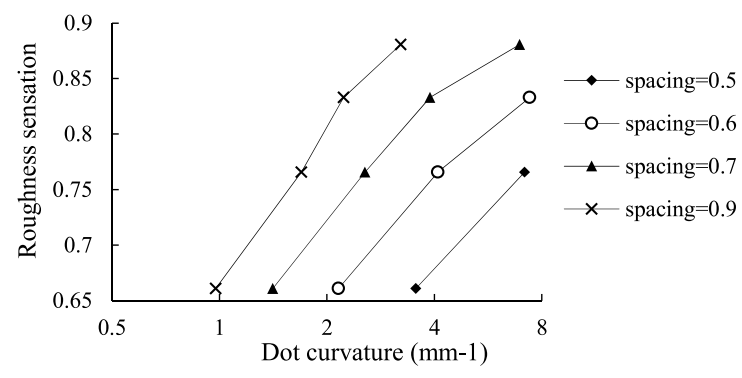

Figure 10: Relationship between the dot curvature and roughness sensation expected from contour lines

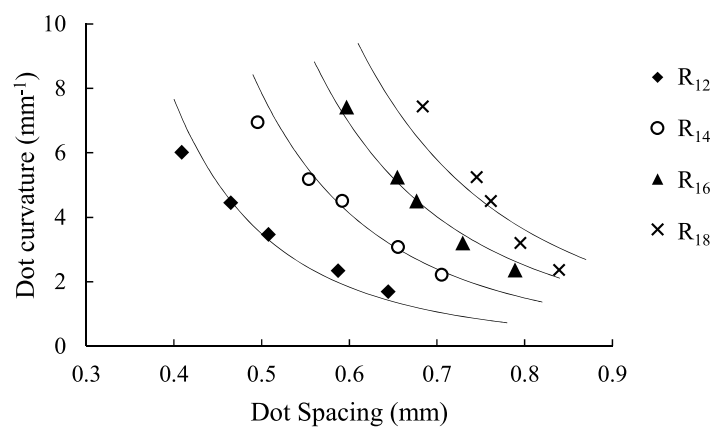

Figure 11: Comparison of contour lines drawn using estimation equations and experimental values

results in Figures 9 and 10 should be parallel straight lines, whereas the upper spacing is narrower than the lower spacing in Figure 9 and there is a slightly curved tendency in Figure 10. In Figure 7b presenting logarithmic values, the contour lines are not parallel and the inclination increases with the roughness sensation. This suggests that the balance between the effect of the dot spacing and dot curvature is not always constant but varies depending on the magnitude of roughness sensation or the range of the dot spacing or dot curvature. In this way, when examined in detail, the magnitude of the roughness sensation is not a simple linear sum and a more complicated approximate surface is required to improve the estimation accuracy. However, considering the accuracy of the values used for estimation, such as the accuracy of the quantification of the roughness sensation and the large individual differences in the experimental result, further detailed examination is considered difficult. 
We next consider the relevance of the present result to previous results. Many previous studies have investigated the perception of roughness by tactile receptors, and response characteristics of each receptor to a time series of change in stimulation have been clarified. Tanaka et al. [16] conducted an experiment in which the amplitude and frequency of an oscillator applied to the finger were changed and they found that the roughness sensation peaked at the sensitivity frequencies of the Meissner and Pacinian corpuscles. The peak frequencies vary depending on the participant (i.e., depending on whether the roughness sensation peaked at the sensitivity frequency of the Meissner corpuscle, the sensitivity frequency of the Pacinian corpuscle, or both frequencies), and there is thus an individual difference in the judgment of roughness.

Tanaka et al. [17] developed a tactile sensor with a PVDF film having a high-frequency piezoelectric effect similar to the response of tactile receptors. They applied this sensor to the tactile evaluation of cloth [18] and showed that the magnitude of the power spectral density for frequencies from 20 to $100 \mathrm{~Hz}$ (i.e., wavelengths from 0.75 to $3.75 \mathrm{~mm}$ ) corresponding to the response of the Meissner corpuscle has a strong correlation with the sensory evaluation of roughness. As an approach based on numerical calculation, Shirado et al. [19] clarified the distribution of the strain energy generated in the skin during a touch by adopting the finite element method. The temporal and spatial distribution patterns at the position of the tactile receptors showed that low-frequency information is detected by the Merkel corpuscle, the shape pattern is detected by the Meissner corpuscle, and the high-frequency roughness and frictional vibration is detected by the Pacinian corpuscle. In this model, when the input frequency is changed in the range of several tens of Hertz to $100 \mathrm{~Hz}$ corresponding to the experimental range in this paper, the response of the Meissner corpuscle linearly decreases with respect to the logarithm value of the input frequency.

Considering the experimental results obtained in the present study from the viewpoint of a time series of the change in stimulation, the stimulus frequency is proportional to the reciprocal of the dot spacing. Therefore, reversing the coefficients in Equation (1) suggests that the roughness sensation will linearly decrease with respect to the logarithmic value of the frequency. This is in agreement with the response characteristic of the Meissner corpuscle in the above analysis. Although it is difficult to quantitatively evaluate, the dot curvature is thought to affect the amplitude of vibration through deformation of the skin surface. It is therefore estimated that each term in Equation (1) corresponds to the frequency component and the amplitude component of the physical input to the tactile receptors.

As described above, although error due to the linear approximation is included, in the present experimental range, the approximate shape of roughness sensation can be expressed in the form of the linear sum of the logarithmic values of the dot curvature and dot spacing.

\subsection{Examination of individual differences}

In the sensory evaluation results, individual differences were observed in the amount of change in the dot spacing with respect to the dot curvature. There were also participants whose answers were irregular. Cluster analysis was thus performed for all answers, and the participants were classified into four groups. Contour lines for the average value by group are shown in Figure 12. The shape of a contour line varies depending on the group. Group A (eight participants) is considered to have a small change in dot spacing with respect to a change in curvature, Group C (five participants) is considered to have a large change in dot spacing with respect to a change in curvature, and group B (10 participants) is considered to be intermediate of groups A and C. Participants in Group D (seven participants) gave irregular answers (i.e., the order of roughness sensation was often reversed among the series of samples for these participants). It is considered that the criterion for determining the roughness sensation is not stable in this group. The differences among groups $\mathrm{A}, \mathrm{B}$, and $\mathrm{C}$ are thought to be the parameter weightings. Group A can be said to be spacing oriented in that the dot
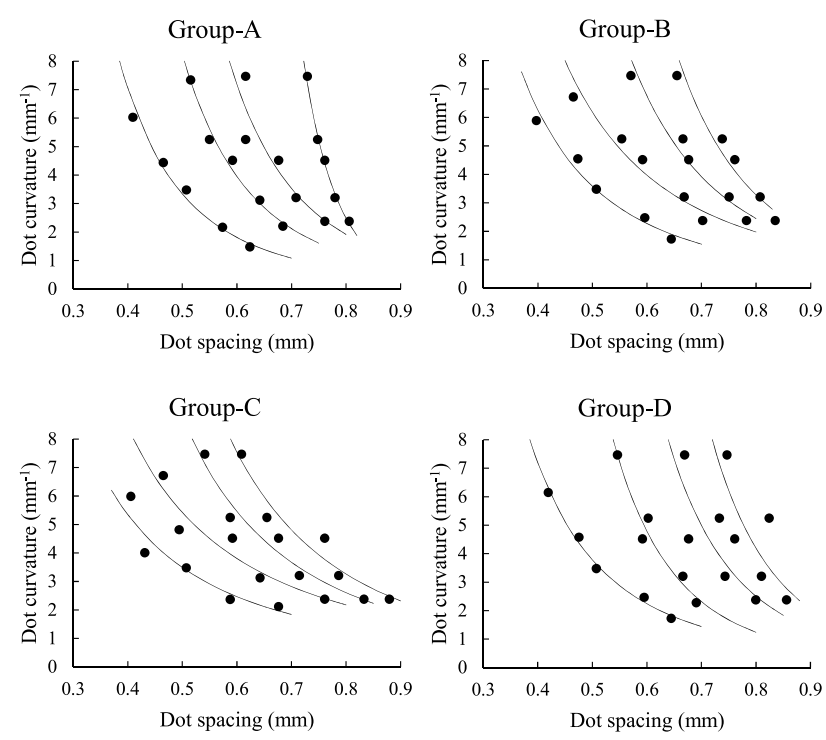

Figure 12: Comparison of contour lines among participant groups 
Table 2: Regression coefficients for participant groups

\begin{tabular}{|c|c|c|c|}
\hline & Group-A & Group-B & Group-C \\
\hline Dot spacing & 1.02 & 1.02 & 1.01 \\
\hline Dot curvature & 0.23 & 0.33 & 0.42 \\
\hline Constant term & 0.86 & 0.80 & 0.75 \\
\hline
\end{tabular}

spacing is emphasized in the process of evaluating roughness sensation while group $\mathrm{C}$ can be said to be shape oriented in that dot curvature is emphasized. Group B is intermediate of groups $\mathrm{A}$ and $\mathrm{C}$. The ratio of participants is about $30 \%$ spacing oriented, about $30 \%$ intermediate, about $20 \%$ shape oriented, and the rest unstable. Hence, even with the same dot pattern, it is considered that the roughness is perceived according to different criteria by participants, which may lead to individual differences in higher tactile evaluation.

To express such differences numerically, we adopt multiple regression of the roughness sensation in the same way as it was used in the previous section for the average value of each group. The coefficients of terms are given in Table 2. The coefficient of dot curvature increased in the order of group $\mathrm{A}<\mathrm{B}<\mathrm{C}$ while the coefficient of dot spacing were about 1 in each group. These results suggest that participants in group $\mathrm{C}$ place more importance on dot curvature when determining the roughness sensation than do participants in group A. This difference may be due to individual differences in the ability to detect differences in curvature.

We did not repeat trials in our experiment and we therefore cannot judge whether the differences seen among participants are unique to the participants or due to variations in the trials.

Factors unique to the participant include physical properties of the skin that affect the sensory receptive mechanism (e.g., the skin thickness, fingerprint, and skin flexibility), the frequency band used in evaluating roughness as clarified by Tanaka et al., and the method of touching (with the contact state affected by the contact force and speed of movement). The present paper focused on the detection sensitivity and thus did not specify the touching method, but the effects of dimensional parameters on the roughness sensation may depend on the contact force and speed of movement. Future work needs to investigate the cause of the differences observed among participant groups.

\subsection{Scope of application of the experimental results}

The scope of application of the experimental results is discussed below from the viewpoint of the activity of touching and sensory modality.
The effect of the frequency of stimulation on the roughness sensation depends on whether the touching method is active touching (i.e., moving a finger) or passive touching (i.e., moving the sample). When the speed of movement is constant, the difference in sensation between active touching and passive touching is considered small [20]. However, as the speed varies, the roughness sensation changes according to the speed in passive touching while the same roughness is perceived in active touching [21]. This is thought to be due to the fact that the change in the vibration power with speed is corrected by the self-reception sensation in the case of the active touch. In this paper, we constrained only the finger used in the experiments and the direction of touch, and the scope of the results is thus limited to active touching. In passive touching, not only the effect of the dot spacing but also the effect of the dot curvature may be affected by the speed of movement. Therefore, when conducting the same experiment with passive touching, there is the possibility that there will be a bias depending on the contact force and speed in the pattern of contour lines.

\section{CONCLUSION}

Participants selected sets of samples that presented the same roughness sensation from many types of samples made with a $3 \mathrm{D}$ printer. We drew contour lines of the roughness sensation and investigated the distribution of the roughness sensation with respect to the dot spacing and dot curvature from the pattern of contour lines in the range of $0.4-0.9 \mathrm{~mm}$ and dot curvature in the range of $2-8 \mathrm{~mm}^{-1}$. The obtained results are summarized as follows.

(1) Multiple sets of dot curvature/dot spacing with equivalent roughness sensation were obtained, and we drew contour lines of the roughness sensation in the two-dimensional parameter space.

(2) We quantified the contour lines and found that the logarithmic value of the dot curvature and the magnitude of roughness sensation have a linear relationship. We obtained a multiple regression equation that estimates the roughness sensation from logarithmic values of dot spacing/dot curvature.

(3) There were large individual differences in the shape of contour lines, and it was found that the weighting of the dot curvature varies depending on the participant. Participants could be divided into three groups according to the weighting of the dimensional parameters. 
The present study clarified the magnitude of the roughness sensation generated by a combination of the dot spacing and dot curvature. It is considered that the findings obtained in this paper can be used as design guidelines for dot patterns and similar textures.

\section{ACKNOWLEDGEMENTS}

Part of this work was supported by MEXT KAKENHI (Grant-in-Aid for Scientific Research (B), No. JP16H02888).

\section{REFERENCES}

1. Okamoto, S., Nagano, H., and Yamada, Y.; Psychophysical dimensions of tactile perception of textures, IEEE Transactions on Haptics, 6(1), pp.81-93, 2012.

2. Ban, A., Tamuraya, M., Takeuchi, T., et al.; Interior parts for vehicles, Japan patent P5253103, 2013. (in Japanese)

3. Yonehara, M., Tatsuno, J., Nakamura, H., Takehara, S., Fujii, T., and Fukumoto, T.; Effect of gradational patterns on positional accuracy of active touch, Ergonomics, 49(6), pp.279-288, 2013. (in Japanese)

4. Kawasegi, N., Fujii, M., Shimizu, T., Sekiguchi, N., Sumioka, J., and Doi, Y.; Evaluation of the human tactile sense to microtexturing on plastic molding surfaces, Precision Engineering, 37(2), pp.433-442, 2013.

5. Kawasegi, N., Fujii, M., Shimizu, T., Sekiguchi, N., Sumioka, J., and Doi, Y.; Physical properties and tactile sensory perception of microtextured molded plastics, Precision Engineering, 38(2), pp.292-299, 2014.

6. Kawasegi, N., Fujii, M., Shimizu, T., Sekiguchi, N., Sumioka, J. and Doi, Y.; Tactile sensory control on a plastic molding surfaces fabricated by micromachining - Characterization of various types of texture-, Journal of the Japan Society for Precision Engineering, 80(7), pp.692-698, 2014. (in Japanese)

7. Aizawa, J., Hiraide, S., Kitano, A., Kashiyama, T., and Kamijo, M.; Visual and tactile evaluation on roughness sensation of plastic texture for injection mold design, Kansei Engineering, 14(4), pp.457-464, 2015. (in Japanese)

8. Aizawa, J., Kashiyama, T., and Kamijo, M.; Relationship between contact condition and roughness sensation of plastic texture for injection mold design, Kansei Engineering, 16(1), pp.163-169, 2017. (in Japanese)

9. Shimojo, M., Maeno, T., Shinoda, H., and Sano, A.;
Tactile recognition mechanism and applied technology (augmented version), S\&T Publishing, Tokyo, pp.1215, 2014. (in Japanese)

10. Noro, K., Inukai, S., and Saito, S.; An analysis of individual differences on sensory measurement of roughness, Ergonomics, 12(6), pp.227-234, 1976. (in Japanese)

11. Noro, K.; Individual Information process in feeling measurement of roughness of turned surface, Journal of the Japan Society for Precision Engineering, 43(5), pp.530-535, 1977. (in Japanese)

12. Mishima, I., and Nakano, K.; Analysis of sensory surface roughness based on mechanical measurement, Journal of the Japan Society for Precision Engineering, 42(12), pp.1102-1108, 1976. (in Japanese)

13. Yanagisawa, H., and Takatsuji, K.; A Kansei evaluation method for surface texture considering with prior visual expectation, Transactions of the Japan Society of Mechanical Engineers, Series (C), 78(796), pp.3830-3841, 2012. (in Japanese)

14. Yanagisawa, H., and Takatsuji, K.; A method for extraction of visual expectation effect on surface texture (A case of visual expectation effect on tactile roughness perception of plastic texture), Transactions of the Japan Society of Mechanical Engineers, Series (C), 79(807), pp.4028-4038, 2013. (in Japanese)

15. Aizawa, J., and Takizawa, R.; On characteristics of fine dot shape formed by 3D printer, Research Reports of Nagano Prefecture General Industrial Technology Center, 13, pp.51-55, 2018. (in Japanese)

16. Tanaka, M., Tsuchimi, D., and Okuyama, T.; Mechanism of haptic perception - Influence of amplitude and frequency for smoothness -, Proceedings of the Tohoku University Global Center of Excellence Programme, pp.368-377, 2012.

17. Tanaka, Y., Tanaka, M., and Chonan, S.; Development of a sensor system for collecting tactile information, Transactions of the Japan Society of Mechanical Engineers, Series (C), 72(724), pp.3818-3825, 2006. (in Japanese)

18. Tanaka, Y., Tanaka, M., and Chonan, S.; Measurement of tactile sensation using sensor system for collecting tactile information, Transactions of the Japan Society of Mechanical Engineers, Series (C), 73(727), pp.817-824, 2007. (in Japanese)

19. Shirado, H., Konyo, M., and Maeno, T.; Modeling of tactile texture recognition mechanism, Transactions of the Japan Society of Mechanical Engineers, Series (C), 73(733), pp.2514-2522, 2007. (in Japanese) 
20. Lederman, S. J.; The perception of surface roughness by active and passive touch, Bulletin of the Psychonomic Society, 18(5), pp.253-255, 1981.

21. Yoshioka, T., Craig, J.C., Beck, G.C., and Hsiao, S. S.; Perceptual constancy of texture roughness in the tactile system, Journal of Neuroscience, 31(48), pp.17603-17611, 2011.

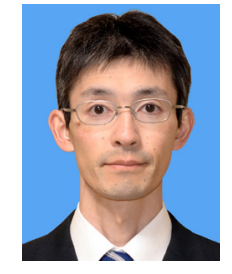

Junpei AIZAWA (Member)

Junpei Aizawa is a Senior Researcher of Nagano Prefecture General Industrial Technology Center, Japan. He received the B.E. and the M.E. degrees in Engineering from Keio University, in 1996 and 1999, respectively, and the Ph.D. degree in Engineering from Shinshu University, in 2019. His research interests include Kansei measurement and precision measurement. He is a member of Japan Society of Kansei Engineering and The Japan Society for Precision Engineering.

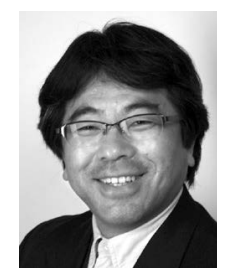

Masayoshi KAMIJO (Member)

He received the Dr. Eng. degree from Shinshu University in 1995. He is currently a professor in interdisciplinary graduate school of science and technology of Shinshu University. He is engaged in research on measurement and assessment system on human health and comfort.

E-mail:kamijo@shinshu-u.ac.jp 